\title{
EFEITOS DE UM PROTOCOLO FISIOTERAPÊUTICO PARA TRATAMENTO DE PACIENTE COM DISFUNÇÃO TEMPOROMANDIBULAR: UM RELATO DE CASO
}

\author{
EFFECTS OF A PHYSIOTHERAPEUTIC PROTOCOL FOR TREATMENT OF \\ PATIENTS WITH TEMPOROMANDIBULAR DYSFUNCTION: A CASE REPORT
}

Rafaela Mendonça Amoras; Raissa Rodrigues Pereira Lima; Gabriela Trindade Felix; Wiviane Maria Torres de Matos Freitas

\section{RESUMO}

A articulação temporomandibular desenvolve funções essenciais, como fonação, mastigação, deglutição e respiração. As alterações e manifestações dolorosas da ATM podem ocorrer tanto no local, como em músculos e articulações que estão interligadas com a articulação, como da coluna cervical e cintura escapular. Sendo assim, o objetivo do estudo analisar os efeitos de um protocolo de tratamento fisioterapêutico na disfunção temporomandibular. Para isso, foi realizada avaliação fisioterapêutica, constituída por um questionário, exame físico e avaliação funcional. Os objetivos e evoluções mostraram estabelecimento de um protocolo de tratamento. A $1^{\underline{a}}$ fase de tratamento objetivou a analgesia, por meio de técnicas de terapia manual e eletroterapia. A $2^{\text {a }}$ fase visou aumento de amplitude de movimento, com mobilizações e alongamentos, já a $3^{a}$ fase consistia em adaptação à nova prótese. $\mathrm{Na}$ reavaliação atestou-se melhoras nos itens "presença de zumbido, dores de cabeça frequentes, dores na face/pescoço, dor na mastigação, dor ao abrir a boca ao máximo, cansaço nos músculos e uso de placa interoclusal", assim como melhora significativa no índice temporomandibular. Portanto, foi possível constatar que o protocolo terapêutico se mostrou eficiente para a paciente, sendo capaz de diminuir o quadro álgico e a tensão muscular, ganhando amplitude de movimento e funcionalidade. Ressalta-se a necessidade da atuação multidisciplinar para a reabilitação integral da paciente.

Palavras chaves: Transtornos da ATM, Articulação Temporomandibular, Fisioterapia.

\begin{abstract}
The temporomandibular joint is more important, such as phonation, chewing, swallowing and breathing. Painful changes and manifestations of TMJ can occur both at the site, and in muscles and joints that are interconnected with the joint, such as the cervical spine and scapular girdle. Therefore, the study was analyzed as an instrument of physiotherapeutic treatment in temporomandibular dysfunction. For this, a physical therapy review was performed, determined by a questionnaire, physical examination and functional evaluation. Objectives and evolutions are a treatment protocol. 1st phase of treatment with the objective of analgesia, through techniques of manual therapy and electrotherapy. The 2nd phase was aimed at the range of motion, with mobilizations and stretching, already in the 3rd phase of adaptation to the new prosthesis. The reevaluation was better documented in the "presence of tinnitus, frequent headaches, pain in the face / neck, pain in chewing, pain when opening a
\end{abstract}


mouth to the max, muscles can be used and the use of interocclusal plaque", as well as the significant without temporomandibular relation. Therefore, it was possible to verify that the therapeutic protocol was efficient for the patient, being able to reduce the pain and muscular tension, gaining range of movement and functionality. The need for multidisciplinary action for the integral rehabilitation of the patient is emphasized.

Descriptors: Temporomandibular Joint Disorders, Temporomandibular Joint, Physical Therapy Specialty.

\section{INTRODUÇÃO}

A articulação temporomandibular (ATM) é uma das articulações mais utilizadas pelo homem e que faz parte do sistema estomatognático, sendo constituída por uma unidade biológica ou morfofuncional que está localizada anatomicamente no território crânio-cérvico-facial ${ }^{1,2}$.

A ATM é uma articulação sinovial do tipo gínglimo modificada, as faces articulares envolvidas nela são compostas pelo côndilo da mandíbula na parte inferior, na parte superior pelo tubérculo articular e a fossa mandibular. Os ligamentos que compõe a ATM são os colaterais, capsular, esfenomandibular e estilomandibular, os músculos, podem ser divididos em elevadores (masseter, temporal, pterigóideo medial) e abaixadores da mandíbula (pterigóideo lateral, digástrico, milo-hióideo, gênio-hióideo) ${ }^{3}$.

Atua como responsável pelas funções de mastigação, deglutição, fonoarticulação e respiração. Trata-se de um sistema complexo composto por ossos, músculos, ligamentos e dentes, e, além disso, sua mobilidade está relacionada não apenas pelos músculos mastigatórios, mas por uma sinergia entre os numerosos músculos que participam também de outras ações voluntárias e reflexas ${ }^{1,2}$.

Para que ocorra o funcionamento adequado da ATM, a própria articulação, a oclusão dental e o equilíbrio neuromuscular devem relacionar-se harmonicamente, portanto o equilíbrio mandibular relaciona-se também com o equilíbrio muscular corporal $^{3}$.

E cabe destacar, que se o funcionamento não for adequado, os músculos mastigatórios e a ATM podem sofrer lesões e serem alvos de um grupo de doenças, que quando reunidas, geram a disfunção temporomandibular (DTM), podendo ser 
classificada em dois grandes subgrupos que consistem nas de origem articular (atrogênica) e nas de origem muscular (miogênica). Por ter uma etiologia multifatorial, a DTM pode ocorrer por fatores estruturais, neuromusculares, oclusais (perdas dentárias, desgaste dental, próteses mal adaptadas, cáries, restaurações inadequadas entre outras), psicossomático (devido a tensão há um aumento da atividade muscular que gera espasmo e fadiga), hábitos parafuncionais (bruxismo, onicofagia, apoio de mão na mandíbula, sucção digital ou de chupeta) e lesões traumáticas ou degenerativas da ATM ${ }^{4}$.

As alterações e manifestações dolorosas da ATM podem ocorrer localmente na articulação ou à distância destas. Devido o envolvimento de diversos músculos, as repercussões da DTM se estendem para além de alterações relacionadas às funções da articulação, podendo estar associada às desordens posturais, principalmente relacionadas a rotação e inclinação da cabeça e da cintura escapular².

Essa relação entre a ATM e as alterações posturais de cabeça, cervical e ombro, é explicada pelo fato de a biomecânica da coluna cervical e da cintura escapular estar interligadas através de um sistema neuromuscular comum. Por isso, o realinhamento e as compensações corporais podem causar perturbações na função e organização da ATM, sendo que o contrário também é verdadeiro ${ }^{5}$.

Nesse contexto, para que o tratamento da DTM seja completo e efetivo é essencial à atuação de uma equipe multiprofissional trabalhando em conjunto, incluindo cirurgiões-dentistas, fisioterapeutas, fonoaudiólogos, além de psicólogos, otorrinolaringologistas, neurologistas e clínicos da dor, para que seja realizada uma avaliação ampla e conjunta com a finalidade de encontrar os fatores causais das disfunções, a partir dos achados clínicos pertinentes a cada área de atuação 4 .

No que tange a atuação da fisioterapia, essa tem o intuito de evitar a necessidade de realização de cirurgia e como objetivos: reposicionar a mandíbula, minimizar a dor muscular, diminuir a inflamação, melhorar a amplitude de movimento da ATM, reduzir a carga da articulação, melhorar a postura e fortalecer o sistema musculoesquelético facial e postural ${ }^{4}$. Spillere e Rosas ${ }^{6}$ afirmam que a fisioterapia dispõe de vários recursos no tratamento da disfunção da ATM, dentre elas a massoterapia, cinesioterapia e eletroterapia, proporcionando, além do alívio da sintomatologia, o restabelecimento da função do aparelho mastigatório e da postura. 
Diante do exposto, o objetivo deste estudo foi analisar os efeitos da aplicação de um protocolo de tratamento fisioterapêutico para paciente com disfunção temporomandibular.

\section{METODOLOGIA}

Trata-se de um relato de caso de intervenção, descritivo de delineamento retrospectivo, a partir de prontuário. A paciente pertencia ao atendimento ambulatorial do estágio, as sessões aconteciam na Clínica Escola de Fisioterapia do Centro Universitário do Pará (CESUPA), localizada na Rua João Balbi no 1327, bairro São Brás - Belém - Pará - Brasil, CEP 66060-270. No período de fevereiro a maio de 2019.

Após assinatura do TCUD (Termo de compromisso de Utilização de Dados), foram extraídos do prontuário os dados previamente coletados por meio de uma ficha de avaliação fisioterapêutica, por meio de um questionário de 19 perguntas, elaborado pela professora responsável pelo estágio de fisioterapia em traumatologia.

Da ficha de avaliação foram coletadas dados da identificação e analisadas as limitações funcionais descritas pela paciente, avaliação postural, alteração da abertura da boca, ausculta da ATM para verificar se havia presença de ruídos, crepitação e estalido, palpação muscular, goniometria cervical, índice temporomandibular (Fricton e Shiffman 2008), que avalia a funcionalidade da ATM e se tem presença de dor durante o movimento. Esta avaliação foi aplicada no início do tratamento, anteriormente à aplicação do protocolo, e reaplicada ao final da $2^{\mathrm{a}}$ fase do protocolo.

A partir da leitura das evoluções e dos objetivos terapêuticos, foi possível perceber o protocolo estabelecido para tratamento fisioterapêutico da DTM, que pôde ser dividido em três fases (Tabela 1). Este treinamento teve duração de 14 semanas sendo realizadas duas sessões semanais, em um total de dez sessões com duração de 50 minutos cada. 
Tabela 1 - Protocolo de tratamento

\begin{tabular}{|c|c|c|}
\hline FASE & OBJETIVOS & RECURSOS/TÉCNICAS \\
\hline $\begin{array}{l}\text { Fase } 1 \\
5 \text { sessões } \\
50 \text { minutos } \\
\text { cada }\end{array}$ & - Diminuir a dor; & $\begin{array}{l}\text {-Ultrassom: parâmetros F: 1MHZ, T: } 2 \text { min, 0,7 m/cm², } \\
\text { contínuo; } \\
\text { - Pompage (músculos: elevador da escápula, trapézio } \\
\text { fibras descendentes e esternocleidomastóideo); } \\
\text { - Massagem terapêutica } \\
\text { - Tração cervical }\end{array}$ \\
\hline $\begin{array}{l}\text { Fase } 2 \\
5 \text { sessões } \\
50 \text { minutos } \\
\text { cada }\end{array}$ & $\begin{array}{l}\text { - Aumentar a amplitude } \\
\text { de movimento; } \\
\text { - Fortalecer os músculos } \\
\text { faciais; } \\
\text {-Restabelecer a } \\
\text { funcionalidade; }\end{array}$ & $\begin{array}{l}\text { - Mobilização articular passiva; } \\
\text { - Distração mandibular; } \\
\text { - Dessensibilização de pontos-gatilho; } \\
\text { - Massagem terapêutica; } \\
\text { - Exercícios funcionais de movimentos mandibulares; } \\
\text { - Facilitação neuromuscular proprioceptiva; } \\
\text { - Alongamento ativo; } \\
\text { - Exercício ativo e ativo-resistido; } \\
\text { - Exercício de consciência corporal. }\end{array}$ \\
\hline $\begin{array}{l}\text { Fase } 3 \\
5 \text { sessões } \\
50 \text { minutos } \\
\text { cada }\end{array}$ & $\begin{array}{l}\text {-Adaptar a prótese de } \\
\text { forma funcional; } \\
\text {-Manter a funcionalidade }\end{array}$ & $\begin{array}{l}\text { - Exercícios funcionais de movimentos mandibulares; } \\
\text { - Alongamento ativo; } \\
\text { - Exercício ativo e ativo-resistido; } \\
\text { - Exercício de consciência corporal; } \\
\text { - Isostretching. }\end{array}$ \\
\hline
\end{tabular}

Todos os dados foram submetidos à análise estatística descritiva e os valores de amplitude de movimento e intensidade de dor inicial e final foram comparados para analisar a eficácia do tratamento fisioterapêutico na disfunção temporomandibular posterior à execução de um protocolo. Além disso, foram comparados os dados qualitativos como a tensão muscular, presença de dor e limitação funcional.

\section{RESULTADO}

Das duas avaliações realizadas, uma foi feita no início da fase 1 e outra no final da fase 2, como descrito na tabela 2. Para a realização da terceira fase, seria necessária a confecção de uma nova prótese para a paciente, entretanto, as evoluções sinalizaram que não houve a troca da mesma. Sendo assim, não foi 
possível realizar a adaptação da prótese na terceira fase, os registros evidenciaram manutenção dos objetivos da fase anterior.

Tabela 2 - Dados de avaliação e reavaliação

\begin{tabular}{|c|c|c|}
\hline FICHA DE AVALIAÇÃO & AVALIAÇÃO & REAVALIAÇÃO \\
\hline Sente dor na ATM? & Sim, lado esquerdo & $\begin{array}{l}\text { Sim, lado direito e } \\
\text { esquerdo }\end{array}$ \\
\hline Apresenta ruído na ATM? & Sim, lado esquerdo & Sim, lado esquerdo \\
\hline Tem dor nos ouvidos? & Não & Não \\
\hline Apresenta zumbido nos ouvidos? & Sim, lado esquerdo & Não \\
\hline Tem dores de cabeça freqüentes? & $\begin{array}{l}\text { Sim, na região } \\
\text { temporal }\end{array}$ & Não \\
\hline $\begin{array}{l}\text { Tem dores na face ou no pescoço (dores } \\
\text { irradiadas)? }\end{array}$ & $\begin{array}{l}\text { Sim, no músculo } \\
\text { trapézio }\end{array}$ & Não \\
\hline Aperta os dentes durante o dia? & Sim & Sim \\
\hline Suas articulações doem quando mastiga? & Sim & Não \\
\hline $\begin{array}{l}\text { Suas articulações doem quando abre a boca ao } \\
\text { máximo? }\end{array}$ & Sim & Não \\
\hline $\begin{array}{l}\text { Sente cansaço nos músculos da face após longa } \\
\text { refeição? Onde? }\end{array}$ & $\begin{array}{l}\text { Sim, no músculo } \\
\text { masseter }\end{array}$ & Não \\
\hline Faz uso de placa nos dentes (placa interoclusal)? & Sim & Não \\
\hline Faz uso de prótese? & Sim & Sim \\
\hline Você é uma pessoa: & $\begin{array}{l}\text { Ansiosa, tensa, } \\
\text { nervosa }\end{array}$ & Ansiosa, tensa, nervosa \\
\hline
\end{tabular}

Fonte: Prontuário, 2019.

Foi observado, na avaliação do exame físico, que a paciente apresentava desvio da abertura de boca para a esquerda e presença de crepitação na ausculta do lado esquerdo. Sobre a avaliação postural foi registrado: cabeça anteriorizada e em rotação para a esquerda, ombros protusos e o ombro esquerdo mais elevado.

Com relação a palpação muscular, a paciente apresentava dor nos músculos masseter, pterigóideo medial pterigóideo lateral, esternocleidomastoideo e trapézio, 
assim como apresentou tensão muscular aumentada. Na reavaliação do exame físico, postural e palpação a paciente não foram constadas melhoras tão significativas.

Em relação ao índice temporomandibular de Fricton e Shiffman, é possível verificar na tabela 3, que na segunda fase houve evolução significativa da paciente em relação à primeira fase.

Tabela 3 - Dados da relação ao índice temporomandibular de Fricton e Shiffman.

\begin{tabular}{|c|c|c|c|c|}
\hline \multirow[b]{2}{*}{ ÍNDICE FUNCIONAL } & \multicolumn{2}{|c|}{ AVALIAÇÃO } & \multicolumn{2}{|c|}{ REAVALIAÇÃO } \\
\hline & $\begin{array}{l}\text { Amplitude de } \\
\text { movimento }\end{array}$ & $\begin{array}{l}\text { Dor durante } \\
\text { movimentação }\end{array}$ & $\begin{array}{l}\text { Amplitude de } \\
\text { movimento }\end{array}$ & $\begin{array}{l}\text { Dor durante } \\
\text { movimentação }\end{array}$ \\
\hline $\begin{array}{l}\text { Máxima abertura da } \\
\text { boca ativa sem dor } \\
(>40 \mathrm{~mm})\end{array}$ & $10 \mathrm{~mm}$ & (1) & $30 \mathrm{~mm}$ & (0) \\
\hline $\begin{array}{l}\text { Máxima abertura da } \\
\text { boca ativa }(>40 \mathrm{~mm})\end{array}$ & $\begin{array}{l}\text { Não } \\
\text { conseguiu } \\
\text { realizar }\end{array}$ & (1) & $30 \mathrm{~mm}$ & (0) \\
\hline $\begin{array}{l}\text { Máxima abertura da } \\
\text { boca } \\
(>40 \mathrm{~mm})\end{array}$ & $\begin{array}{l}\text { Não } \\
\text { conseguiu } \\
\text { realizar }\end{array}$ & (1) & $40 \mathrm{~mm}$ & (0) \\
\hline $\begin{array}{l}\text { Desvio lateral direito } \\
(>7 \mathrm{~mm})\end{array}$ & $\begin{array}{l}\text { Não } \\
\text { conseguiu } \\
\text { realizar }\end{array}$ & (1) & $10 \mathrm{~mm}$ & (0) \\
\hline $\begin{array}{l}\text { Desvio lateral } \\
\text { esquerdo }(>7 \mathrm{~mm})\end{array}$ & $\begin{array}{l}\text { Não } \\
\text { conseguiu } \\
\text { realizar }\end{array}$ & (1) & $15 \mathrm{~mm}$ & (0) \\
\hline Protusão (>7mm) & $\begin{array}{l}\text { Não } \\
\text { conseguiu } \\
\text { realizar }\end{array}$ & (1) & $5 \mathrm{~mm}$ & (0) \\
\hline $\begin{array}{l}\text { Superposição vertical } \\
\text { dos incisivos }\end{array}$ & $\begin{array}{l}\text { Não } \\
\text { conseguiu } \\
\text { realizar }\end{array}$ & (1) & $\begin{array}{l}\text { Não } \\
\text { conseguiu } \\
\text { realizar }\end{array}$ & (1) \\
\hline
\end{tabular}

Legenda: (0) - Sem dor

(1) - Com dor

Fonte: Prontuário, 2019.

\section{DISCUSSÃO}

A DTM envolve um grande número de problemas clínicos que afetam os músculos mastigatórios, ATM e estruturas associadas. Por apresentar origem multifatorial, os principais sinais e sintomas dessa disfunção se encontram não só na 
região da ATM, mas também acomete regiões adjacentes ${ }^{7}$. Os resultados apresentados demonstram que, antes do tratamento, a paciente apresentava dores musculares, causadas por hipertonia dos músculos esternocleidomastóideo, trapézio superior, dores na cabeça e no ouvido.

Um estudo realizado com 117 indivíduos analisou a correlação de transtornos temporomandibulares (TTM), identificou que 50,9\% apresentavam grau leve de TTM, $21,8 \%$ grau moderado e $0,9 \%$ grau severo, constatou que destes que apresentavam grau moderado ou severo, correspondendo a $30,8 \%$, mais da metade possuíam alguma alteração na posição da cabeça e ainda que $88 \%$ tinham alterações nos ombros $^{5}$. Da mesma forma, na avaliação postural deste estudo foi evidenciado anteriorização e rotação de cabeça, ombros bilateralmente protusos, além de elevação de ombro unilateral, corroborando com a literatura encontrada.

Em razão da interligação entre essas estruturas, hábitos como posição de dormir, estresse ou conflitos emocionais, podem repercutir na funcionalidade dessas articulações, que pode ter sido o caso da paciente, pois as evoluções evidenciavam que a mesma apresentava questões emocionais que associadas às alterações de oclusão, causavam maior tensão nos músculos mastigatórios e adjacentes (trapézio superior, esternocleidomastóideo) ${ }^{8}$.

Por tal, não cabe afirmar que o tratamento da DTM feito exclusivamente pelo fisioterapeuta implicará na melhora da condição geral da paciente, uma vez que é necessária a atuação do profissional odontólogo em conjunto com a fisioterapia. Este fato pôde ser observado neste estudo, pois após o protocolo de tratamento, não houve melhora significativa da dor à palpação e nem das tensões musculares, devido à falta da adequação da prótese dentária e a condição oclusal que a paciente se encontrava. Pois a fisioterapia alcançou objetivos importantes, porém a musculatura e a articulação sofrem adaptações que se não estiver com uma prótese adequada, irá provoca dor na região maxilar, pode ocasionar feridas e permanência da tensão e da dor nos músculos mastigatórios e adjacentes, além de presença de alterações posturais 9 .

Em um estudo com uma amostra de 27 indivíduos, com diagnóstico clínico de DTM, foi analisada a mobilidade articular e avaliação dos músculos envolvidos diretamente com a $\mathrm{ATM}^{10}$, e este trabalho confirma os mesmos achados avaliativos 
de tendência à hipomobildade articular, bem como presença de espasmos e pontos gatilhos na maioria dos músculos mastigatórios, além de dor na mobilidade articular.

Sendo assim é necessário lançar mão de recursos que objetivem o alívio dos sintomas, restabelecendo a função normal do aparelho mastigatório da paciente. No presente estudo, foram incluídos no processo de reabilitação técnicas de terapia manual, exercícios de alongamentos musculares, mobilizações articulares e exercícios para estabilização segmentar cervical, o que é afirmado como técnicas eficazes nesse processo por Pelicioli, Florianovicz e Batista ${ }^{11}$. Já que a fisiologia e objetivos desses métodos e técnicas é provocar o relaxamento muscular, aumento da amplitude de movimento e reorganização do trabalho muscular.

Cabe ressaltar que o mau posicionamento da coluna cervical pode influenciar diretamente na relação entre o maxilar superior e a mandíbula, se fazendo assim necessário a inclusão do tratamento da coluna cervical no plano terapêutico. Neste estudo, a intervenção proposta para a coluna cervical foi a aplicação de massagem terapêutica, dessensibilização de pontos em gatilho e cinesioterapia, as técnicas mostraram grande contribuição para a recuperação da função mandibular ${ }^{12}$.

Em uma revisão de literatura sobre a desativação de pontos-gatilho para o tratamento da dor miofascial, foram selecionadas as técnicas que incluem a utilização de fármacos, técnicas de fisioterapia convencional e de agulhamento seco ou com injeção de substâncias anestésicas, demonstrando alta eficiência no alcance deste objetivo $^{13}$. Da mesma forma, neste estudo foi realizada a aplicação da fisioterapia convencional, utilizando recursos terapêuticos manuais, percebendo que houve relevante redução da dor miofascial da paciente a cada fase.

\section{CONCLUSÃO}

Diante do exposto, é possível constatar que a aplicação de um protocolo terapêutico se mostrou eficiente no tratamento da DTM. Lançando mão de recursos como cinesioterapia, massagem e eletroterapia, são possíveis restabelecer a função do paciente com DTM, diminuindo o quadro álgico e de tensão muscular, ganhando amplitude de movimento. No entanto, ainda se percebe a necessidade a atuação de uma equipe multidisciplinar para minimizar os sintomas, facilitando o tratamento fisioterapêutico e a reabilitação integral da paciente. 


\section{REFERÊNCIAS}

1. Garcia JD, Oliveira AAC. A fisioterapia nos sinais e sintomas da disfunção da articulação temporomandibular (ATM). Hórus 2017;6(1):111-22

2. Santos BF, Fragelli TBO. Prevalence of temporomandibular joint disorders and neck pain in musicians: a sytematic review. Fisioter Mov 2017 Oct/Dec;30(4):839-48

3. Freitas DG, Pinheiro ICO, Vantin K, Meinrath NCM, Carvalho NAA. Os efeitos da desativação dos pontos-gatilho miofasciais, da mobilização articular e do exercício de estabilização cervical em uma paciente com disfunção temporomandibular: um estudo de caso. Fisioter. Mov. 2011 Jan/Mar;24(1):33-38.

4. Donnarumma M, et al. Disfunções temporomandibulares: sinais, sintomas e abordagem multidisciplinar. Rev CEFAC 2010;12(5):788-94.

5. Chaves PDJ, Oliveira FEMD, Damázio LCM. Incidence of postural changes and temporomandibular disorders in students. Acta ortopedica brasileira 2017;25(4): 162164.

6. Spillere A, Rosas RF. Tratamento fisioterapêutico na disfunção da articulação temporomandiular (ATM) um estudo de caso. Tese [Graduação em fisioterapia]. 2010 7. Assis TO, Matheus SS, Marcio MV. O uso do laser na reabilitação das desordens temporomandibulares. Fisioter Mov 2012 Abr/Jun;25(2):453-59.

8. Biasotto-Gonzalez DA, et al. Correlação entre disfunção temporomandibular, postura e qualidade de vida. Journal of Human Growth and Development 18.1 (2008): 79-86.

9. Torres F, Campos LG, Filipini HF, Weigert LK, Vecchia GFD. Efeitos dos tratamentos fisioterapêutico e odontológico em pacientes com disfunção temporomandibular 2017. Fisioterapia em Movimento;25(1).

10. Souza MLA. Avaliação biomecânica em indivíduos portadores de desordem temporomandibular. 2015. Monografia (Graduação em Fisioterapia) - Universidade Estadual da Paraíba; 2015.

11. Pelicioli M, Myra RS, Florianovicz VC, Batista JS. Tratamento fisioterapêutico nas desordens temporomandibulares. Rev Dor 2017 Out/Dez;18(4):355-61.

12. Tosato JP, Biasotto-Gonzalez DA. Avaliação pré e pós-tratamento fisioterapêutico na disfunção temporomandibular. Fisioterapia Brasil 2006 Mar/Abr;7(2):159-61. 
13. Culpi M, Martinelli ABMC. Desativação de pontos-gatilho no tratamento da dor miofascial. Revista Brasileira de Medicina de Família e Comunidade 2018;13(40). 